\title{
Crystalization-enhanced Stability by Effectively Suppressing Pho-tooxidation Defect for Lasing Emission and Electroluminescent Device
}

\section{He Zhang}

Nanjing University of Posts and Telecommunications

Dongqing Lin

Nanjing University of Posts and Telecommunications

Huifang Liu

Nanjing University of Posts and Telecommunications

Wei Liu

College of Optical, Mechanical and Electrical Engineering, Zhejiang A\&F University Hangzhou 311300, Zhejiang, China

\section{Yang-Cheng Wang}

Institute of Advanced Materials (IAM) Nanjing University of Posts \& Telecommunications (NUPT) 9

Wenyuan Road Nanjing 210046, Jiangsu, China

\section{Dong Jin}

Institute of Advanced Materials (IAM) Nanjing University of Posts \& Telecommunications (NUPT) 9 Wenyuan Road

Zhu-Xin Li

State Key Laboratory of Bioelectronics, School of physics, Southeast University, Nanjing 210096, Jiangsu, China

\section{Xinwen Zhang}

Nanjing University of Posts and Telecommunications

\section{Lei Huang}

Institute of Advanced Materials (IAM) Nanjing University of Posts \& Telecommunications (NUPT) 9 Wenyuan Road Nanjing 210046, Jiangsu, China

\section{Shasha Wang}

Nanjing University of Posts and Telecommunications

\section{Chunxiang Xu}

Southeast University https://orcid.org/0000-0001-8116-2869

\section{Linghai Xie ( $\nabla$ iamlhxie@njupt.edu.cn )}

Centre for Molecular Systems and Organic Devices (CMSOD) \& Key Laboratory for Organic Electronics and Information Displays \& Institute of Advanced Materials (IAM) \& Jiangsu National Synergetic Innov https://orcid.org/0000-0001-6294-5833 
Article

Keywords:

Posted Date: December 13th, 2021

DOI: https://doi.org/10.21203/rs.3.rs-1122306/v1

License: (c) (i) This work is licensed under a Creative Commons Attribution 4.0 International License. Read Full License 


\section{Abstract}

Effectively protection against photooxidation of wide bandgap semiconductors remains a challenge that become the bottleneck on the road to commercialization. Herein, we demonstrated the crystallization effects of fluorene-based blue emitters on long-wavelength green band (g-band) defect with the welldefined organic micro/nanocrystals obtained via the self-assembly of surfactant-assisted reprecipitation methods. For the key molecule model 2,2'-bi(9,9-dipropyl)fluorene (DDC3F), the self-assembled nanorods film ex-hibits an ultrastable spectral stability without the presence of g-band emission $(530 \mathrm{~nm})$, and even main-tains the deep-blue emission under ultraviolet exposure $(\sim 3 \mathrm{~h})$. In contrast, the spin-coated amorphous film shows a poor color purity with a green-index of $\sim 4$. Our results offer a robust evidence on the princi-ple in aggregate-enhanced stability, which is severely ignored by the general application of amorphously glassy molecules in OLED devices. In addition, the single nanorod as Fabry-Pérot (FP) optical resonator exhibits size-dependent dual wavelength lasing at $392 \mathrm{~nm}$ (threshold: $102 \mathrm{~mW} / \mathrm{cm} 2$ ) and $412 \mathrm{~nm}$ (threshold: $216 \mathrm{~mW} / \mathrm{cm} 2$ ), and generates the deep-blue electroluminescence in solutionprocessing OLED devices. The supramolecular self-assembled micro/nanocrystals strategy provides a potential platform to maintain ultrastable color purity in wide-bandgap optoelectronic device.

\section{Introduction}

Organic wide-bandgap semiconductors (OWBGS) are the fascinating and promising materials with a numerous of potential application, such as flexible electronics, ${ }^{1-3}$ organic light-emitting diodes (OLEDs), ${ }^{4,5}$ organic solid-state lasers (OSSLs), ${ }^{6,7}$ organic photovoltaics (OPVs), ${ }^{8,9}$ nanogenerators ${ }^{10,11}$ as well as memristive neurocomputing, ${ }^{12,13}$ even artificial intelligence. ${ }^{14}$ In the past 30 years, the thirdgeneration OWBGS have received wide attention due to their advantages over atom-precise synthesis and superior performances. Compared with inorganic counterparts such as $\mathrm{GaN}^{15,16} \mathrm{SiC},{ }^{17,18} \mathrm{ZnO},{ }^{19}$ as well as diamonds, however, the essential breakthrough of the third-generation OWBGS has not been made in terms of molecular design, structure-property relationships, and photoelectronic device applications. It is significant but still elusive to excavate the inherent rules and deeply investigate the association between molecular structures, aggregate morphologies and optoelectronic performances of OWBGS. Salbeck groups have successfully introduced spirobifluorenes to suppress crystalline state and afford high electroluminescence performances in OLED devices, which offer a key cornerstone for a disruptive and transformative paradigm of amorphous and glassy molecular states. ${ }^{20}$

Since 1990s 2000s, fluorene-based building blocks have been indispensable candidates in OWBGSbased systems and photoelectronic devices. ${ }^{21,22}$ Generally, amorphous molecules with high glassy transition temperature $(T g)$ serve as the stable active layers, where molecular arrangements are disordered and loose. In this case, the generation of massive defects from the $\mathrm{O}_{2} / \mathrm{O}$. intrusion in air environment (Fig. 1A) is detrimental to luminescence efficiency, stability, color purity as well as poor reproducibility in photoelectronic devices. ${ }^{23,24}$ In fluorene-based OWBGS systems, defects are generally originated from the presence of ketone sites and aggregation behaviors, where the former has been 
demonstrated but is rather difficult to be overcome. ${ }^{25,26}$ In order to stabilize the fluorene-based semiconductors, various strategies have been explored, including rational molecular design, ${ }^{27}$ aggregate modulation, ${ }^{28}$ device packaging ${ }^{29}$ and so on. For example, in terms of electronic structures, the highest occupied molecular orbital (HOMO) energy level should not be high to defend $\mathrm{H}_{2} \mathrm{O} / \mathrm{O}_{2}$ attacking. On this basis, in terms of molecular structures, aryl substituents are more stable than that of alkyl groups, which plays a key role of steric hindrance in suppressing molecular packing. ${ }^{30}$ Furthermore, bilateral side chain and chain encapsulation in macrocycles also offer a novel method to improve stabilization. However, the mechanisms of tailoring stabilization based on aggregated thin film and other solid states are still unclear and have some contradiction due to some facts.

Recently, we created the intriguing self-assembly of OWBGS into organic micro/nanocrystals through synergistically molecular attractor-repulsor theory (SMART). ${ }^{31,32}$ We previously discovered that the crystalline nanosheets affords the effect of crystal-induced luminescence and/or stability enhancement (CLOSE) on rac-tactic terfluorenes, while the absence of CLoSE effect in meso-configurational terfluorenes (maintaining amorphous state) results in the g-band. ${ }^{33,34}$ Inspired by this, we used such crystalline strategy to create "molecular packing wall" that favors the suppression of oxidation process and chemical ketone defects (Fig. 1B).

Herein, we investigate the generalization of aggregate-enhanced stability and demonstrate the effect of crystallization on preventing photoodixation behaviors, through the blue-emitting model molecule 9,9,9',9'tetrapropyl-9H,9'H-2,2'-bifluorene ( $\left.\mathrm{DDC}_{3} \mathrm{~F}\right)$. The spin-coated amorphous film (as the control state) was found to exhibit a low spectral stability with the presence of $g$-band emission $(530 \mathrm{~nm})$ and the $I_{\text {green }} / I_{\text {blue }}$ value up to 4 under UV irradiation $(3 \mathrm{~h}$ ). In contrast, the 1D nanorods self-assembled via reprecipitation methods show an ultrastable deep-blue emission with an ultrahigh photoluminescence quantum yield (PLQY) of $87 \%$ and a high spectral stability under the same irradiation conditions, suggesting that the photooxidation defect is effectively suppressed. On this basis, the single nanorod as a Fabry-Pe'rot (FP) optical resonator shows a size-controlled dual wavelength lasing at $392 \mathrm{~nm}$ (threshold: $102 \mathrm{~mW} / \mathrm{cm}^{2}$ ) and $412 \mathrm{~nm}$ (threshold: $216 \mathrm{~mW} / \mathrm{cm}^{2}$ ), respectively. Finally, an inverted crystalline OLED device with solution processing in three layers is fabricated with a low turn-on voltage of $3.7 \mathrm{~V}$.

\section{Results And Discussion}

We selected the 9,9-propyldiarylfluorene dimer $\left(\mathrm{DDC}_{3} \mathrm{~F}\right)$ as the model building block to examine the role of crystallization in improving photostability (Figs. S1 and S2) The bulk-shaped single crystal was luckily obtained via the facile volatile process under the conditions of THF/ethanol mixed solvents (1:3 volume ratio) (Fig. S3). According to the single-crystal data, the $\mathrm{DDC}_{3} \mathrm{~F}$ crystal exhibits the monoclinic space group of P 21/c, with corresponding lattice parameters of $a=9.034$ (3) $\AA, b=16.378$ (5) $\AA, c=10.570$ (4) $\AA, \alpha=90^{\circ}, \beta=103.037(7)^{\circ}, Y=90^{\circ}$ (Supplementary Table S1). By further study the difluorenyl backbone exhibits anti-conformation, where the torsional angle between two fluorenyl moieties is approximate to $180^{\circ}$. The propyl chains are oriented on two sides of difluorene plane, which shows massive sites of Van 
der Waals force in symmetric distribution (Fig. S6). Based on above conformational feature, the intermolecular packing of $\mathrm{DDC}_{3} \mathrm{~F}$ building blocks were performed in a herringbone manner with the angle of $58.57^{\circ}$. In such packing architecture, multiple $\mathrm{C}-\mathrm{H} . . . \pi$ intermolecular interactions (in the distance of $2.895 \AA$ ) between two sterically adjacent molecules connecting the alkyl chains and the fluorene planes. In addition, compared with other pendant groups, the propyl chains also possess the role of steric hindrance to increase the distance $(8.29 \AA$ and $8.98 \AA$ ) between two adjacent difluorene plane (Fig. S7). ${ }^{30,35}$

Then, we investigated the surfactant assisted self-assembly of $\mathrm{DDC}_{3} \mathrm{~F}$ into micro/nanocrystals to explore its crystalline features. Figs. 2A, 2B and 2D show the scanning electron microscopy (SEM) image, transmission electron microscopy (TEM) image and fluorescence microscopy of the self-assembled 1D nanorods with smooth surfaces and sharp edges. The length $(\mathrm{L})$ of nanorods is about $20-40 \mu \mathrm{m}$ and the width $(W)$ are around $600-1200 \mathrm{~nm}$, with the length: width ratio as $\approx 100: 3$. And atomic force microscope (AFM) image reveals that the average thickness of nanorods is about $90 \mathrm{~nm}$ (Fig. 2E). Besides, XRD spectra of the nanorods show a series of sharp diffraction peaks corresponding to the $\{011\}$ crystal plane (Fig. 2F). The observation of high order diffraction peaks of (011), (022) indicate the high crystallinity of these assembled nanorods that adopt a lamellar nanostructure with the (011) crystal plane adhered to the Si substrate. Meanwhile, the corresponding selected area electron diffraction (SAED) pattern were further used to corroborate the crystalline property of nanorod architectures, where the detected directions are parallel to [011 crystal direction and perpendicular to the flat nanorod surface of (011) single plane, respectively (Fig. 2C). Along the [100] crystal direction, the SAED spots confirm the single crystal state of nanorod architectures, where the red rounded sets of SAED spots are assigned to $(100)$ and $(0 \overline{1} 1)$ crystal planes with d-spacing values of $8.80 \AA$ and $8.72 \AA$, respectively. These SAED patterns are consistent with the XRD feature and are further reconfirmed by the simulation results (inset of Fig. 2F). In addition, the - crystal planes (011) and ( 011 ) on the top and bottom surfaces, the (100), (100), (0 1 1) and (011) crystal planes on the lateral surfaces, which are completely correspondence with theoretically predicted growth morphology (Fig. S8). On this basis, we investigated the crystalline effect on the photophysical properties and photo-stability of $\mathrm{DDC}_{3} \mathrm{~F}$. Fig. $3 \mathrm{~A}$ shows the normalized steady-state absorption and PL spectra of $\mathrm{DDC}_{3} \mathrm{~F}$ in different states. Two absorption band centered at 270 and $330 \mathrm{~nm}$ were observed on the dilute solution and the spin-coated amorphous film, the latter of which is assigned to the $\pi-\pi^{*}$ transition on difluorenyl moiety. For $\mathrm{PL}$ spectra, $\mathrm{DDC}_{3} \mathrm{~F}$ in the dilute solution exhibits a clearly vibronic progression of 0-0, 0-1, 0-2 and 0-3 transitions at $373,388,408$ and $438 \mathrm{~nm}$, respectively. The $\mathrm{DDC}_{3} \mathrm{~F}-$ based amorphous film exhibits a slightly red-shifted with the vibronic progression of $0-0,0-1,0-2$ and $0-3$ transitions at $375,390,410$ and $440 \mathrm{~nm}$, respectively, where the relative intensity of $0-0$ emission is diminished. However, the $\mathrm{DDC}_{3} \mathrm{~F}$-based nanorods film only shows 0-1, 0-2 and 0-3 emission peaks at 392, 412 and $443 \mathrm{~nm}$, respectively, according to the fitting of Lorentzian line shapes (Fig. S9), while the 0-0 emission is completely vanished. This deeply-blue emission feature is probably consistent with the highly ordered $\mathrm{H}$-aggregate emission. ${ }^{36}$ Deeply, the photoluminescence quantum yield (PLQY) of $\mathrm{DDC}_{3} \mathrm{~F}$ is $97 \%$ 
in dilute solution, $57 \%$ on amorphous film and $87 \%$ on crystalline nanorods film (Fig. 3B), respectively. These results reveal that such nanorod architectures possess the crystallization-induced luminescence enhancement (CIEE) effect as approximately the highest level of PLQY. ${ }^{37}$ Meanwhile, the corresponding photophysical data were shown in Table 1 and Figs. S10-12. According to the $k_{r}=\Phi_{F} / \tau_{F L}$ equation, the calculated radiative deactivation rate $\left(k_{r}=1.23 \times 10^{9} \mathrm{~S}^{-1}\right)$ of nanorods film is enhanced, while the nonradiative decay rate $\left(k_{n r}=1.83 \times 10^{8} \mathrm{~S}^{-1}\right)$ is reduced compared to that of $D D D C_{3} F$ in amorphous film. This is probably because of the inhabitation of quenching effect in crystal through the introduction of pendant propyl-alkyl chains.

The thermostability and photostability were examined for spin-coating amorphous film and drop-casting nanorods film. Initially, $\mathrm{DDC}_{3} \mathrm{~F}$ powder displays excellent thermostability with a high thermal decomposition temperature of $320^{\circ} \mathrm{C}$ (Fig. S5). After thermal annealing at $100^{\circ} \mathrm{C}$ under ambient conditions for 10 min $\sim 3 \mathrm{~h}$, the PL spectra of both film states are maintained without the addition of green emission band, indicating the excellent spectra stability. It is noted that, probably owing to the presence of crystallization behaviors, the intensity of $388 \mathrm{~nm}$ (0-0 band) on amorphous film were reduced (Fig. S13). Further, phtotostability of films was investigated under UV irradiation (room temperature, RH: $70 \%$, light: $80 \mathrm{~W}$ ). In Fig. 3C, after UV exposure for $10 \mathrm{~min}$, the amorphous film generates a new green emission band at 500-600 nm. Further exposed for 1 3 h, such green emission at $530 \mathrm{~nm}$ is gradually enhanced, with the green index $I_{\text {green }} / I_{\text {blue }}$ increasing from 1 to 4 . These results indicate the presence of photooxidation process on amorphous film, which affords oxidative defects and results in the spectra instability (Top of Fig. 3C). In contrast, the nanorods film is capable of maintaining deep-blue emission (with $I_{\text {green }} / I_{\text {blue }}=0$ ), almost without the generation of green-emission band even under the same or longer UV irradiation (Fig. 3D). The excellent photostability was also confirmed by the patterning experiment through brushing process, as shown in Fig. S14. Under the same UV exposure, the crystalline nanorod region "CMSOD" can still maintain the blue-emission, while the amorphous background region shows the yellow-green luminescence.

To gain insight into the photostability mechanism of nanorods film, FT-IR spectra and transient spectra were used to detect the oxidative defects (Fig. 3E). We observed that the FT-IR spectrum of amorphous film (undergoing UV irradiation) generates an infrared absorption at $1720 \mathrm{~cm}^{-1}$, assigned to the stretching vibration of $\mathrm{C}=0$ on ketone moieties. Meanwhile, the lifetime at $530 \mathrm{~nm}$ emission was measured as $\sim 5.4 \mathrm{~ns}$, confirming the ketone emission with partly forbidden transition feature (Fig. 3F). ${ }^{38}$ In contrast, no $\mathrm{C}=0$ stretching vibration (at $\sim 1720 \mathrm{~cm}^{-1}$ ) was observed on nanorod films after the same UV exposure, along with no emission behaviors with the lifetime of $\sim 5 \mathrm{~ns}$, both of which indicate the nonexistence of ketone defects. These results reveal that the ketone defects during the exudation process are majorly formed on the amorphous film, while the nanorod film has a high-efficient influence on suppressing the oxidation process for the formation of ketone defects (Fig. S15). Therefore, the closely molecular packing in crystalline nanorod architectures, analogous to molecular wall guard, allows the excellent ability to isolate oxygen (in air) and protect the 9-position of fluorenyl moieties from undergoing 
exudation. It is noted that the high photostability from such crystalline molecular wall strategy is significant to efficiency, reproducibility and color purity of photoelectronic devices.

In order to further the verify the strategy of crystalization-enhanced stability, the deep blue emission of $\mathrm{DDC}_{3} \mathrm{~F}$ nanorod as Fabry-Pe'rot (FP) optical resonator was also tentatively examined through micro/nanocrystals nanosecond lasing. In Figs. 4A and S17, the lasing spectra of nanorod architecture with the length of $42.8 \mu \mathrm{m}$ possesses the violet emission at $392(0-1)$ and $412(0-2) \mathrm{nm}$ under the pump density of 187 to $234 \mathrm{~mW} / \mathrm{cm}^{2}$. However, the shorter nanorod structure ( $16 \mu \mathrm{m}$ length) only shows a stimulation emission at $392 \mathrm{~nm}$ (Fig. 4B). According to the variation of output intensity as a function of pump density (Fig. 4C), the lasing threshold was evaluated as $\sim 102 \mathrm{~mW} / \mathrm{cm}^{2}$, and the maximum width at half intensity (FWHM) was calculated as FWHM $=0.14 \mathrm{~nm}$, along with the quality factor Q of 2800 (Fig. $\mathrm{S} 18 \mathrm{~A})$. Moreover, if increasing the nanorod length excessing $\sim 70 \mu \mathrm{m}$, it is surprising that the simulation emission switches into the $412 \mathrm{~nm}$ (0-2 channel) (Fig. 4D), along with the lasing threshold of 216 $\mathrm{mW} / \mathrm{cm}^{2}$ (Fig. 4E), FWHM $=0.46 \mathrm{~nm}$ and the quality factor Q $=896$ (Fig. S18B). In contrast, the amorphous film only exhibited amplified self-emission (ASE) feature at $390 \mathrm{~nm}$, with the pump energy threshold of $\sim 99 \mathrm{~mW} / \mathrm{cm}^{2}$ (Fig. S19). These results reveal that that such simulation emission switching is closely associated with the nanorod architectures of size-dependent from reabsorption effect, ${ }^{35,39}$ which provide the stable platform of efficiently propagating and manipulating miniaturized optics chip on subwavelength scale or microscale.

Finally, we tentatively examined the electroluminescent feature of self-assembled crystalline film based on the inverted OLED configuration of ITO/ZnO $(10 \mathrm{~nm}) / \mathrm{Cs}_{2} \mathrm{CO}_{3}(20 \mathrm{~nm}) /$ Crystals film $(150 \mathrm{~nm})$

Table 1

the photophysical properties of $\mathrm{DDC}_{3} \mathrm{~F}$ in dilute THF solution, amorphous film and nanorods film.

\begin{tabular}{|c|c|c|c|c|c|c|}
\hline $\mathrm{DDC}_{3} \mathrm{~F}$ & $\lambda_{\mathrm{abs}}{ }^{\mathrm{a}}$ & $\lambda_{\mathrm{em}}{ }^{\mathrm{b}}$ & $\Phi_{\mathrm{F}}{ }^{\mathrm{c}}$ & $\tau_{F L}{ }^{d}$ & $k_{r}^{e}$ & $\mathrm{~K}_{\mathrm{nr}}^{\mathrm{f}}$ \\
\hline THF solution & $279,284,330$ & $373,388,408,438$ & 0.95 & 0.79 & $1.20 \times 10^{9}$ & $6.33 \times 10^{7}$ \\
\hline Amorphous film & 300,330 & $375,390,410,440$ & 0.57 & 0.67 & $8.51 \times 10^{8}$ & $6.42 \times 10^{8}$ \\
\hline Nanorods film & $330,348,366$ & $392,412,443$ & 0.87 & 0.71 & $1.23 \times 10^{9}$ & $1.83 \times 10^{8}$ \\
\hline \multicolumn{7}{|c|}{$\begin{array}{l}\text { a Absorption maximum of } \mathrm{DDC}_{3} \mathrm{~F} \text { in three different states (dilute THF solution: } 10^{-5} \mathrm{~mol} / \mathrm{L} \text {, spin- } \\
\text { coating amorphous film and nanorods film). }{ }^{b} \text { Emission maximum. }{ }^{c} \text { Fluorescence quantum yield } \\
\left(\Phi_{\mathrm{F}}\right) \text { determined by a calibrated integrating sphere. }{ }^{d} \text { Fluorescence lifetime. }{ }^{e} \text { Radiative deactivation } \\
\text { rate calculated according to the } \mathrm{k}_{\mathrm{r}}=\Phi_{\mathrm{F}} / \tau_{\mathrm{FL}} \text { equation. }{ }^{f} \text { Nonradiative deactivation rate calculated } \\
\text { according to the } \mathrm{k}_{\mathrm{nr}}=1 / \tau_{\mathrm{FL}}-\mathrm{k}_{\mathrm{r}} \text { equation. }\end{array}$} \\
\hline
\end{tabular}


/NPB $(40 \mathrm{~nm}) / \mathrm{MoO}_{3}(8 \mathrm{~nm}) / \mathrm{LiF}(1 \mathrm{~nm}) / \mathrm{Al}(100 \mathrm{~nm})$ in Figs. 5A and S20. As the nanorods film is too rough to exhibit the electroluminescence performance, we used thermal annealing method to afford the dendritic-like crystalline film (Figs. S21 and S22). The turn-on voltage (VT) was measured as 3.7 V, indicating efficient charge injection into the crystalline device. When the driving voltage is $7.8 \mathrm{~V}$, the luminance of crystalline device is up to $15 \mathrm{~cd} / \mathrm{cm}^{2}$ (Fig. 5B). Besides, the maximum current efficiency of device display is $0.013 \mathrm{~cd} / \mathrm{A}$ at $4.5 \mathrm{~V}$ (Fig. 5C). Fig. 5D shows the corresponding EL spectra under different driving voltages and the emission peak is centered at $445 \mathrm{~nm}$. It is worth mentioning that three layers were processed by the solution method based on crystalline OLED, which totally different from previous reported. ${ }^{40}$ Therefore, the crystalization strategy demonstrated it is a unique technology for electroluminescent properties, which can be exploited for the whole solution OLED displays.

\section{Conclusions}

To summarize, we pioneering propose the crystalization-enhanced stability strategy for suppressing photooxidation defect of fluorene-based OWBGS. The self-assembled nanorods of the fluorene dimer $\mathrm{DDC}_{3} \mathrm{~F}$ exhibit excellent ultraviolet stability. The nanorods film still keeping the deep blue emission under UV irradiation, which efficiently suppress g-band $(530 \mathrm{~nm})$ of photooxidation defect compared with the amorphous film using the unparalleled superiority of molecular dense packing. Besides, the single nanorod shows dual wavelength deep blue emission laser of dependent-size at $392 \mathrm{~nm}$ and $412 \mathrm{~nm}$ with FP optical resonator, respectively. Finally, the inverted crystalline OLED device with solution processing of three layers was fabricated with a low turn-on voltage of $3.7 \mathrm{~V}$. The fabrication of crystalline OLED device based on solution processing technology further confirmed the enormous potential of our crystalization strategy like-molecular wall.

\section{Methods}

All of the reagents and solvents in the experiment were obtained from commercial suppliers and without further purification unless otherwise noted. 2-bromo-9,9-dipropyl-9H-fluorene and 2-(9,9-dipropyl-9Hfluoren-2-yl)-4,4,5,5-tetramethyl-1,3,2-dioxaborolane were synthesized according to the previously reported methods.

\section{Structural and optical characterizations of organic nanorods}

SEM images were obtained by a scanning electron microscopy (Hitachi S-4800) with accelerating voltage of $5 \mathrm{kV}$. TEM images and selected-area electron diffraction (SAED) were obtained from a transmission electron microscopy (JEOL JEM 2010F, accelerating voltage $100 \mathrm{kV}$ ). One drop of the solution was dropped on a carbon-coated copper grid, and the solvent was evaporated at room temperature. Using the Bruker's Dimension Icon Atomic Force Microscope with trapping mode, the AFM images were measured under ambient conditions and the nanorods were dispersed on silicon substrate. The X-ray diffraction (XRD) of samples was performed on a Bruker D8 X-ray diffractometer with Cu Ka radiation $(\lambda=1.54050$ $\AA)$; the operating $2 \theta$ angle ranges from 5 to $30^{\circ} \mathrm{C}$, with a step length of $0.02^{\circ} \mathrm{C}$. The single-crystal data 
was performed at around $298 \mathrm{~K}$ on a Bruker $2000 \mathrm{CCD}$ area detector using graphite-monochromated Mo Ka radiation $(\lambda=0.71073 \AA$ ). All structures were solved by direct methods using a SHELXS-2015 and refined against $F_{2}$ using a SHELXL-2015. The steady-state absorption spectra of the samples were measured by a PerkinElmer Lambda 35 spectrophotometer at room temperature. Meanwhile, the emission spectra were measured using RF-6000 Plus fluorospectrophotometer. Time-resolved fluorescence decay lifetime data were obtained using an Edinburgh FLSP920 lifetime spectrometer with a $375 \mathrm{~nm}$ laser with the time correlated single-photon counting methods (pulse width: $55 \mathrm{ps}$; pulse repetition frequencies: $20 \mathrm{MHz}$ ). Absolute Photoluminescence quantum yield (PLQY) measurement was used for solution, amorphous film and nanorods film sample by using an Edinburgh Instruments FLS-920 in an integrating sphere. The fluorescence microscopy images of nanorods were measured by an inverted fluorescence microscope (FV1000-IX71, Tokyo, Japan).

\section{Amplified spontaneous emission (ASE) and microcrystals laser measurement}

The excitation source is a liquid-nitrogen-cooled laser (Janis ST-500, Microscope Cryostat) with $325 \mathrm{~nm}$ femtosecond pulse laser at a repetition of $1000 \mathrm{~Hz}$ and pulse duration of $150 \mathrm{fs}$. The lasing spectra of the microrods were excited by a focused $325 \mathrm{~nm}$ laser through a confocal $\mu$-PL system (OLYMPUS BX53). The size of light spot was about $20 \mu \mathrm{m}$ in diameter. The luminescent light was collected through an optical multichannel analyzer (Princeton, Acton SP2500i) and the spectral resolution of the spectrometer was $0.167 \mathrm{~nm}$. All measurements were performed at ambient atmosphere. The pump fluence $(P)=$ $\left(P_{0} / f\right) / A$, where $P_{0}$ is pump power, which is measured via pow meter, $f$ is the repetition frequency, and $A$ is light spot area.

\section{The preparation of crystals OLED device}

The ITO substrate was cleaned by acetone, ethanol, and deionized water, subsequently. The amorphous films were prepared by spin-coating with a spin-coating speed of $2000 \mathrm{rpm}$ for $30 \mathrm{~s}$ and an accelerating speed of $500 \mathrm{rpm} / \mathrm{s}$ in toluene solution. Then, the blended film was dried at $60^{\circ} \mathrm{C}$ for $30 \mathrm{~min}$ in a warm table to remove the residual toluene solvent. After that, the crystals film was obtained by annealing at heating stage for $20 \mathrm{~min}$ at $120^{\circ} \mathrm{C}$. The EL spectra and CIE coordinates of the devices were recordedby a spectra-scan PR655 spectrophotometer. The emission area of the devices is $12 \mathrm{~mm}^{2}$. The current-voltage luminescence characteristics were measured using a combination of a keithley source meter (model 2602) and a luminance meter.

\section{Declarations}

\section{Data availability}

The authors declare that the data that support the findings of this study are available within the article and its Supplementary Information files. All other relevant data are available from the corresponding author upon reasonable request. 
Acknowledgements: The project was supported by National Key Laboratory (2009DS690095), the National Natural Science Foundation of China (61604081, 61605090, U1301243), the Natural Science Foundation of the Jiangsu Higher Education Institutions (16KJB430023), Doctoral Fund of Ministry of Education of China (20133223110007), Excellent science and technology innovation team of Jiangsu Higher Education Institutions (2013), Natural Science Foundation of Jiangsu Province, China (BM2012010), the Six Talent Peaks Project of Jiangsu Province (XCL-CXTD-009), Project funded by the Priority Academic Program Development of Jiangsu Higher Education Institutions, PAPD (YX03002), NUPTSF (NY215055, NY215061). Jiangsu Key Laboratory for Chemistry of Low-Dimensional Materials, Huaiyin Normal University, Huaian 223300 (JSKC20022). Program for Postgraduates Research Innovation in University of Jiangsu Province (KYCX20_0744).

Author contributions: L.X., conceived and initiated the study. H.Z contributed to the fabrication of devices and electrical measurements. D.Q., was responsible for SEM and TEM measurements. F.H., and L.H. were responsible for AFM and XRD measurements. W.L. and Z.X., collected the data. C.Y., and J.D. performed theoretical calculation. H.Z wrote the manuscript. X.W., X.C., S.S., and L.H., revised the manuscript, provided guidance, and directed the project.

Competing interests: The authors declare no competing interests.

Additional information

Supplementary information is available for this paper at https://

Reprints and permissions information is available at http://www.nature.com/reprints.

Correspondence and requests for materials should be addressed to L.X., S.S..

\section{References}

1. Qian, Y. et al. Stretchable Organic Semiconductor Devices. Adv. Mater. 28, 9243-9265, (2016).

2. Li, W. et al. Solution-Processed Wide-Bandgap Organic Semiconductor Nanostructures Arrays for Nonvolatile Organic Field-Effect Transistor Memory. Small 14, (2018).

3. Root, S. E., Savagatrup, S., Printz, A. D., Rodriquez, D. \& Lipomi, D. J. Mechanical Properties of Organic Semiconductors for Stretchable, Highly Flexible, and Mechanically Robust Electronics. Chem. Rev. 117, 6467-6499, (2017).

4. Gu, J.-F. et al. Dumbbell-Shaped Spirocyclic Aromatic Hydrocarbon to Control Intermolecular pi-pi Stacking Interaction for High-Performance Nondoped Deep-Blue Organic Light-Emitting Devices. J. Phy. Chem. Lett. 1, 2849-2853, (2010).

5. Park, J. H., Kim, D. Y., Schubert, E. F., Cho, J. \& Kim, J. K. Fundamental Limitations of Wide-Bandgap Semiconductors for Light-Emitting Diodes. ACS Energy Lett. 3, 655-662, (2018). 
6. Zuo, Z. et al. Spiro-substitution effect of terfluorenes on amplified spontaneous emission and lasing behaviors. J. Mater. Chem. C6, 4501-4507, (2018).

7. Jiang, Y. et al. Organic solid-state lasers: a materials view and future development. Chem. Soc. Rev. (2020).

8. Firdaus, Y. et al. Novel wide-bandgap non-fullerene acceptors for efficient tandem organic solar cells. J. Mater. Chem. A 8, 1164-1175, (2020).

9. Gokulnath, T. et al. A wide-bandgap $\pi$-conjugated polymer for high-performance ternary organic solar cells with an efficiency of $17.40 \%$. Nano Energy 89, 106323, (2021).

10. Fan, F. R., Tang, W. \& Wang, Z. L. Flexible Nanogenerators for Energy Harvesting and Self-Powered Electronics. Adv. Mater. 28, 4283-4305, (2016).

11. Chen, A., Zhang, C., Zhu, G. \& Wang, Z. L. Polymer Materials for High-Performance Triboelectric Nanogenerators. Adv.Sci. 7, 2000186, (2020).

12. Yu, Y. et al. 4,5-Diazafluorene-Based Donor-Acceptor Small Molecules as Charge Trapping Elements for Tunable Nonvolatile Organic Transistor Memory. Adv. Sci. 5, 1800747, (2018).

13. Yu, Y. et al. Small-Molecule-Based Organic Field-Effect Transistor for Nonvolatile Memory and Artificial Synapse. Adv. Funct. Mater. 29, 1904602, (2019).

14. Lee, Y. H. et al. Recent advances in organic sensors for health self-monitoring systems. J. Mater. Chem. C 6, 8569-8612, (2018).

15. Cao, Y., Pomeroy, J. W., Uren, M. J., Yang, F. \& Kuball, M. Electric field mapping of wide-bandgap semiconductor devices at a submicrometre resolution. Nat. Elec. 4, 478-485, (2021).

16. Mashiko, H., Oguri, K., Yamaguchi, T., Suda, A. \& Gotoh, H. Petahertz optical drive with wide-bandgap semiconductor. Nat. Phys. 12, 741-745, (2016).

17. Huang, C., Zhang, H. \& Sun, H. Ultraviolet optoelectronic devices based on AlGaN-SiC platform: Towards monolithic photonics integration system. Nano Energy 77, 105149, (2020).

18. Nguyen, T. K., Aberoumand, S. \& Dao, D. V. Advances in Si and SiC Materials for High-Performance Supercapacitors toward Integrated Energy Storage Systems. Small, e2101775, (2021).

19. Shoute, G., Afshar, A., Muneshwar, T., Cadien, K. \& Barlage, D. Sustained hole inversion layer in a widebandgap metal-oxide semiconductor with enhanced tunnel current. Nat. Commun. 7, 10632, (2016).

20. Salbeck, J., Weissörtel, F. \& Bauer, J. Spiro linked compounds for use as active materials in organic light emitting diodes. Macromol. Symp. 125, 121-132, (1998).

21. Xie, L.-H., Yin, C.-R., Lai, W.-Y., Fan, Q.-L. \& Huang, W. Polyfluorene-based semiconductors combined with various periodic table elements for organic electronics. Prog. Polym. Sci. 37, 1192-1264, (2012).

22. Yu, M.-n. et al. Progress in fluorene-based wide-bandgap steric semiconductors. Chinese. J. Polym. Sci. 35, 155-170, (2016).

23. So, F. \& Kondakov, D. Degradation mechanisms in small-molecule and polymer organic light-emitting diodes. Adv. Mater. 22, 3762-3777, (2010). 
24. Gather, M. C., Kohnen, A. \& Meerholz, K. White organic light-emitting diodes. Adv. Mater. 23, 233-248, (2011).

25. Ferreira, G. R. et al. Controlling photo-oxidation processes of a polyfluorene derivative: The effect of additives and mechanism. Mater. Chem. Phys. 146, 212-217, (2014).

26. Cao, Y., Parker, I. D., Yu, G., Zhang, C. \& Heeger, A. J. Improved quantum efficiency for electroluminescence in semiconducting polymers. Nature 397, 414-417, (1999).

27. Kardelis, V., Denk, M. M. \& Adronov, A. Click-Functionalization of a Poly(Tetrazine-co-Fluorene)Conjugated Polymer with a Series of trans-Cyclooctene Derivatives. Angew. Chem. Int. Ed. 60, 29802986, (2021).

28. Lin, Z.-Q. et al. Preparation and Characterization of Polyfluorene-Based Supramolecular $\pi-$ Conjugated Polymer Gels. J. Phys. Chem. C 115, 4418-4424, (2011).

29. Lin, J. et al. Ultrastable Supramolecular Self-Encapsulated Wide-Bandgap Conjugated Polymers for Large-Area and Flexible Electroluminescent Devices. Adv. Mater. 31, e1804811, (2019).

30. Ou, C.-J. et al. Dimerization effect of fluorene-based semiconductors on conformational planarization for microcrystal lasing. J. Mater. Chem. C 5, 5345-5355, (2017).

31. Li, Y. X. et al. SMART Design of a Bulk-Capped Supramolecular Segment for the Assembly into Organic Interdigital Lipid Bilayer-Like (ILB) Nanosheets. Small 14, (2018).

32. Wang, S. S. et al. Variable segment roles: modulation of the packing modes, nanocrystal morphologies and optical emissions. Nanoscale 10, 13310-13314, (2018).

33. Yu, M.-N. et al. Emission Enhanced and Stabilized by Stereoisomeric Strategy in Hierarchical Uniform Supramolecular Framework. Chem 5, 2470-2483, (2019).

34. Zhang, H. et al. Organic Micro-/Nanocrystals of SFX-Based Attractor-Repulsor Molecules with the Feature of Crystal-Induced Luminescence Enhancement. J. Phys. Chem. C125, 6249-6259, (2021).

35. Li, Y.-X. et al. Wide-bandgap organic nanocrystals with high mobility and tunable lasing emission. J. Mater. Chem. C9, 3171-3176, (2021).

36. Sun, Q. et al. Intermolecular Charge-Transfer-Induced Strong Optical Emission from Herringbone HAggregates. Nano Lett. 21, 5394-5400, (2021).

37. Wu, Z. et al. Crystallization-Induced Emission Enhancement of a Deep-Blue Luminescence Material with Tunable Mechano- and Thermochromism. Small 14, e1802524, (2018).

38. Liang, J. et al. Spectral Stability of Polyfluorene-Based Semiconductors. Acta Phys. Chim. Sin. 26, 946-963, (2010).

39. Wang, X. et al. Tunable Near-Infrared Organic Nanowire Nanolasers. Adv. Funct. Mater. 27, 1703470, (2017).

40. An, M. H. et al. Well-Balanced Ambipolar Organic Single Crystals toward Highly Efficient LightEmitting Devices. Adv. Funct. Mater. 30, 2002422, (2020).

\section{Figures}


A

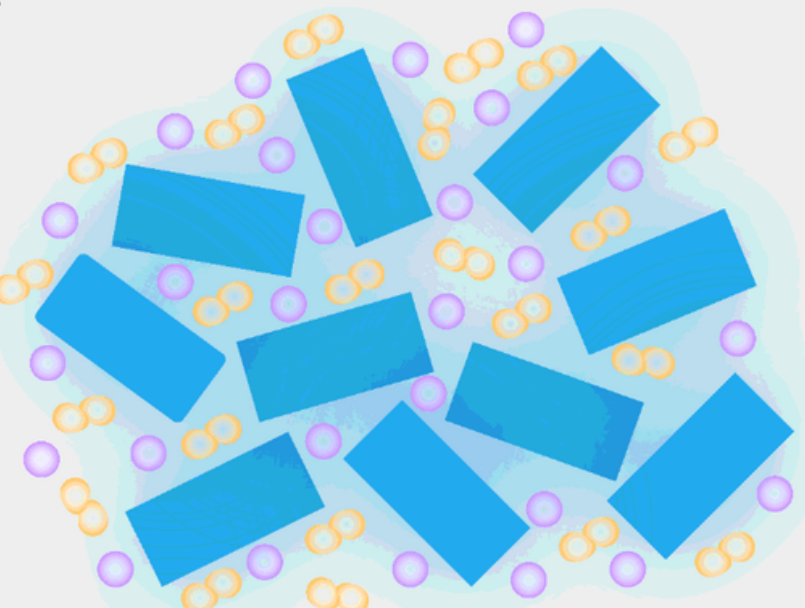

Amorphous

Building block
B

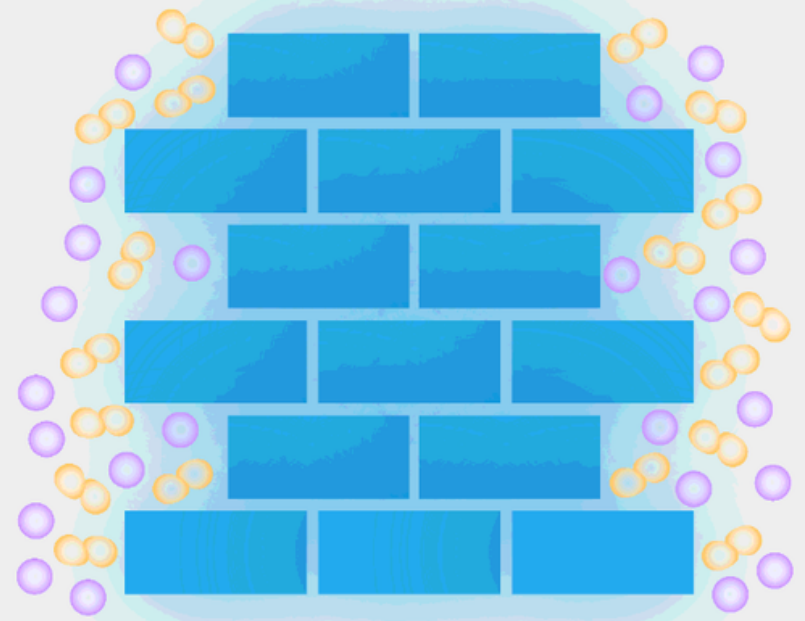

Crystalline

Oxygen $\left(\mathrm{O}_{2}\right)$

Oxygen radical

\section{Figure 1}

Schematic illustration showing the crystallization strategy to suppress the defect of film. (A) Amorphous film with disordered molecules. (B) Micro/nanocrystals film with tightly molecules packing.

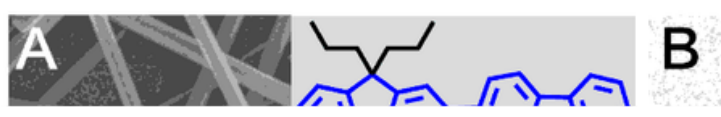

\section{[011] C}

\section{Figure 2}

The self-assembly nanorods microstructure. (A) The SEM image of nanorods grown after $24 \mathrm{~h}$. Inset: the molecule structure of DDC3F. (B) The TEM image of a typical nanorod. (C) The SAED pattern of the 
nanorod and [011] direction as electron beam direction. (D) The fluorescence microscopy image of nanorods excited with UV light (330-380 nm) from a mercury lamp. (E) The AFM image of a nanorod, inset: the height dimension picture. (F) The XRD pattern of nanorods by drop coating on a silicon substrate, inset: a schematic of DDC3F nanorod crystal with the indexed diffraction planes according to crystal structure analysis.

\section{Figure 3}

The photophysical properties and against photooxidation in films. (A) Normalized steady-state absorption and PL spectra of DDC3F in THF solution, amorphous film and nanorods film. (B) The PLQY of DDC3F in three various states measured through an absolute method by using an integration sphere. The PL spectra about UV-irradiation stability of amorphous film (C) and nanorods film (D) with different UV-irradiation times. (E) The FT-IR spectra of pristine sample and different films. And $\mathrm{C}=\mathrm{O}$ stretching frequency is observed at $1720 \mathrm{~cm}-1$ at amorphous film under UV-irradiation. (F) The fluorescence lifetime of amorphous film under UV-irradiation at $530 \mathrm{~nm}$.

\section{Figure 4}

The lasing emission of nanorod. (A) PL spectra obtained from one nanorod excited at different energies at room temperature, inset: the fluorescence microscopy image of one nanorod at $365 \mathrm{~nm}$ UV light and the scale bar is $20 \mu \mathrm{m}$. (B) The PL spectra of laser emission at 0-1 around $392 \mathrm{~nm}$ under different pump densities. (C) Integrated area of the peak $392 \mathrm{~nm}$ as a function of pump density. (D) the PL spectra of laser emission at 0-2 around $412 \mathrm{~nm}$ under different pump densities. (E) Integrated area of the peak 412 $\mathrm{nm}$ as a function of pump density. 
A

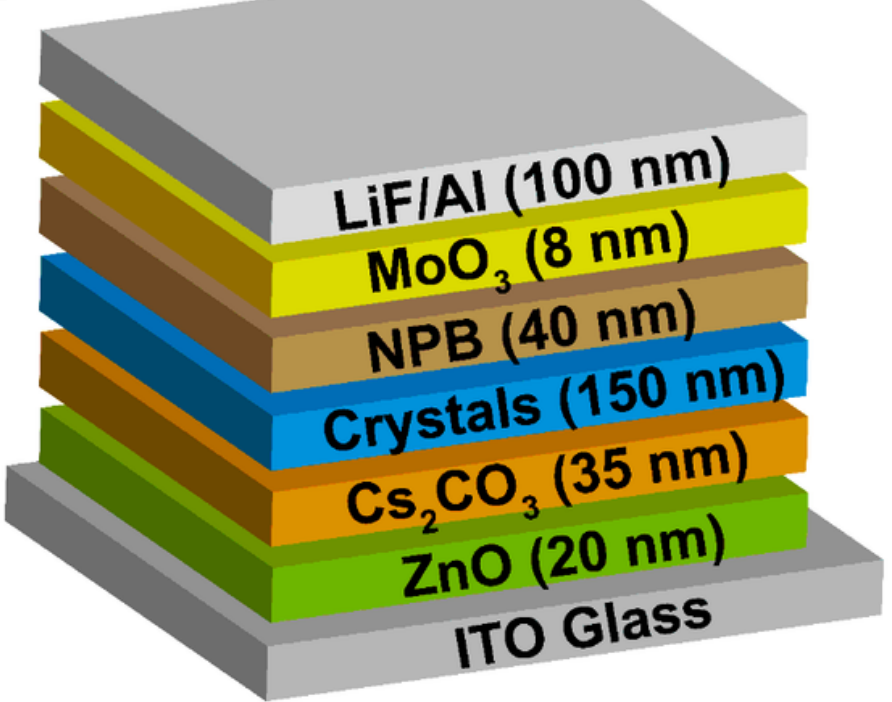

$\mathrm{C}$

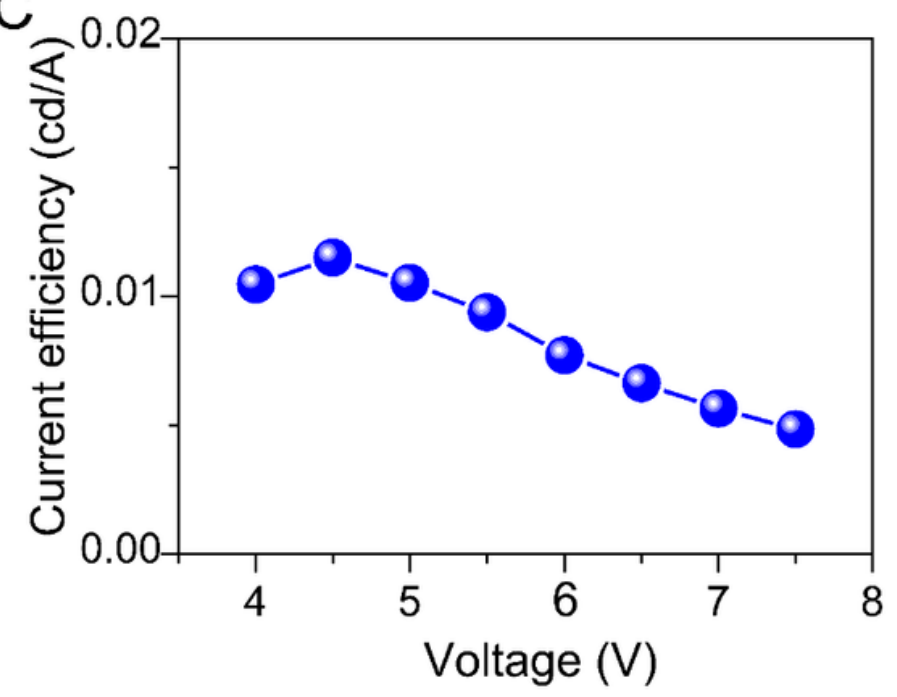

B
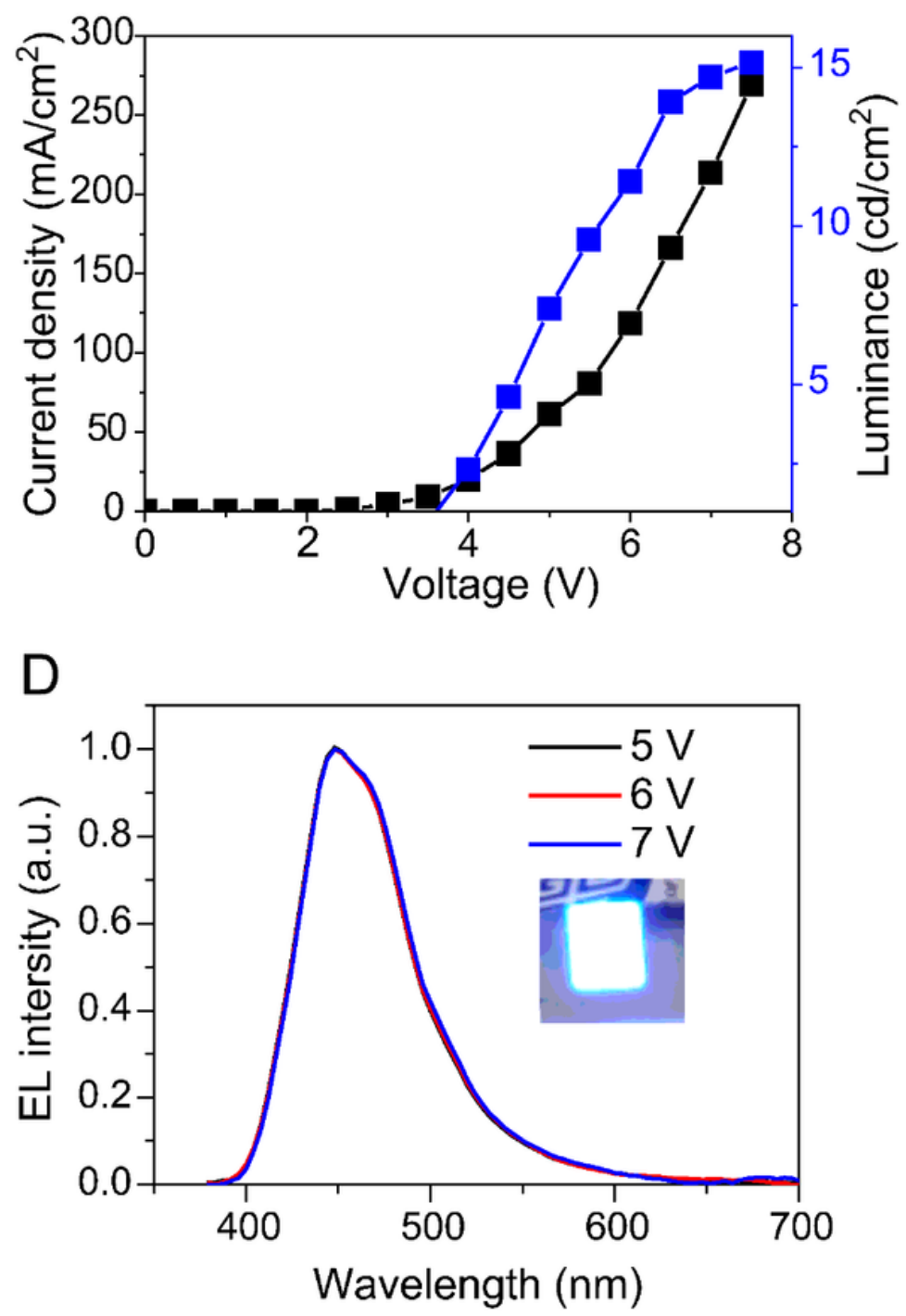

Figure 5

The crystalline OLED Device. (A) The energy level diagram of crystalline OLED with an inverted structure of ITO/ZnO/Cs2CO3/Crystals film /NPB/MoO3/LiF/Al. (B) Current density and luminance at versus driving voltages. (C) Current efficiency characteristics of crystalline OLED. (D) EL spectra of crystalline OLED. Inset: photograph of operating the crystalline OLED at driving voltage of $7 \mathrm{~V}$.

\section{Supplementary Files}

This is a list of supplementary files associated with this preprint. Click to download.

- XieSupportingInformationtoNat.Commun.docx 